\title{
Research on Industrialization Development of Local Cultural Resource Taking Five-Store Bazaar of Jinjiang City as an Example
}

\author{
Hang Ye ${ }^{1}$ Jian Lin ${ }^{1, *}$
}

${ }^{1}$ Tan Siu Lin Business School, Quanzhou Normal University, Quanzhou, Fujian 362000, China

*Corresponding author.Email: 15652473@qq.com

\begin{abstract}
Local cultural resource is not only the premise and foundation for the grand development of cultural industry, but also a new engine for the high-quality development of the regional economy. From the perspective of cultural industry development, this research took the Five-store Bazaar of Jinjiang City, a traditional block as the object, collected data by field visit, questionnaire survey, key interviews and the like methods, and made a systematic analysis on the feasibility and necessity of industrialization development by using "Strength Weakness Opportunity Threats" (SWOT) tool. As a result, a "3 + 2" (i.e., three basic measures including planning adjustment, business expansion, and brand establishment, and two basic principles including differentiation and activation) development model is generated. How to effectively develop cultural and creative industry, fully demonstrate the strength of cultural capital in the region, and avoid falling into the dilemma of low-end homogenization is a major challenge currently faced by the traditional block.
\end{abstract}

\section{Keywords: cultural resource, industrialization development, Five-store Bazaar of Jinjiang City}

\section{INTRODUCTION}

Chinese civilization has a long history, and the profound culture has accumulated a large number and variety of local cultural resources with profound connotation and diverse values to be excavated. The Outline of the National Cultural Development Reform Plan during the "13th Five-Year Plan" Period clearly proposed to "promote the organic integration of cultural resources and industries, expand and guide cultural consumption, and improve the quality and efficiency of cultural industry development". Jinjiang is an important city starting the "Maritime Silk Road Culture", having rich historical relics; it is also a county economic development model in the country and has very strong industrial foundation. Protecting and making good use of local cultural resources and realizing the grand development of the cultural industry is not only a beneficial exploration for practicing the "Jinjiang experience" in the new era, but also the proper meaning of promoting the high-quality development of the "Jinjiang model".

Developed countries have formed a relatively mature model in aspect of the protection and development of local cultural heritages, and have many successful experiences worthy of reference in the legal system, financial security, and public participation. The United States has built an empire of film, media and cultural industries by virtue of its mature business operation experience and rich cultural and creative human resources. The UK pays attention to the economic and social sustainable development of industrial heritage resources development, and emphasizes the joint participation of stakeholders, especially that of local people (Landorf \& Chris, 2009). Japan early put forward the strategy of "building a nation by culture". With the joint efforts of civil society and non-profit organizations, the digitalization industry of cultural resources has been transformed into a pillar industry of the country. Under the influence of Japan, South Korea has built a cultural property development system oriented to people's happiness index. The industrialization development of local cultural resources in China began in the 1990s, and initially depended mainly on regional tourism and sightseeing. In recent years, with the proposal of concepts such as "historical and cultural blocks" and "integration of culture and tourism", the industrialization of local cultural resources has entered a stage of comprehensive development and has become the new focus of local economic and social development. However, there are some common problems such as the single business structure, low industrial integration, and serious homogenization.

The existing cultural resources in the traditional block, Five-store Bazaar of Jinjiang City, are dominated by traditional historical buildings, and are integrated 
into the living inheritance of southern Fujian intangible cultural heritage, having distinct regional characteristics and high industrial development value. At present, there are two methods for studying cultural resources including qualitative and quantitative methods. The former is mainly based on AHP and structural equation model; the latter is mainly based on case analysis and SWOT analysis. Among them, the SWOT method is a relatively systematic and comprehensive research method that makes comprehensive matching and researching judgment on various independent factors such as internal and external advantages, disadvantages, opportunities and challenges in the development of a phenomenon and puts forward applicable development strategies and solutions. Based on the SWOT tool, this research combined with field visits and questionnaire surveys, conducted a comprehensive analysis on the feasibility of the industrialization of cultural resources in the traditional block, Five-store Bazaar of Jinjiang City, and proposed specific measures in order to provide theoretical support and realistic basis for

\section{$2.40 \%$}

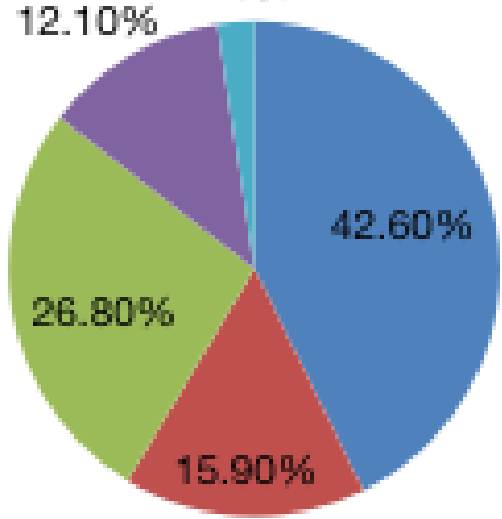

making in-depth research on the industrialization development of the local cultural resources.

\section{RESEARCH AREA AND RESEARCH METHODS}

\section{A. Research area}

The traditional block, Five-store Bazaar of Jinjiang City, is located in the core downtown of Qingyang Street, Jinjiang City, Fujian Province. The existing cultural resources are mainly historical buildings, with the title of "Museum of Ancient Red-brick Houses in South Fujian". In 2019, the scenic spot received 184,900 person-time, accounting for about $58.7 \%$ of the city's number of scenic spot reception, with an year-onyear increase by $81 \%$. There are about 82 commercial points in the block. According to functions, they can be divided into four categories: catering accommodation, tourism and leisure, cultural exhibition transactions, and special cultural and creative products. ("Fig. 1")

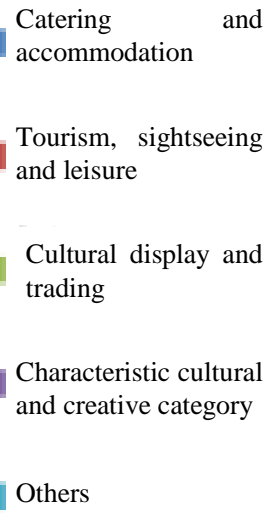

Fig. 1. Distribution of various business types in Five-store Bazaar.

\section{B. Research methods}

Based on literature analysis, the research clarified the internal mechanism of industrialization of local cultural resources. Through field visits, key interviews and questionnaire surveys on tourists, using SWOT tools, the paper proposes specific strategies for the industrialization of cultural resources in Five-store Bazaar.

\section{SWOT ANALYSIS ON INDUSTRIALIZATION DEVELOPMENT OF CULTURAL RESOURCES IN FIVE-STORE BAZAAR OF JINJIANG CITY}

\section{A. Strength analysis}

1) Obvious location strength: Geographically, Jinjiang is located at the corridor hubs of Fuzhou, Quanzhou, Xiamen and Zhangzhou, with convenient transportation. Socially, it keeps a close connection with Taiwan in fields including academic exchange and business trades. Economically, Jinjiang as China's county-level economic leader has outstanding industrial strengths and a cluster of well-known brands.

2) Profound cultural deposits: The Central Plains Culture, Minyue Culture, Marine Culture, Overseas Chinese Culture, and Religious Culture gather here and form a diverse and integrated local cultural resource.

Especially since the reform and opening up, the people of Jinjiang have overcome difficulties and stood up to the tide, making the spiritual core of "love to struggle, dare to win and be kind to others" become synonymous of Jinjiang culture in the new era. 


\section{B. Weakness analysis}

1) The development model has shallow hierarchy, and business types in the block is relatively traditional: First of all, the characteristic business types are not outstanding, and there is homogeneous competition. In the existing business structure of the block, catering and accommodation category accounts for a large proportion, while characteristic cultural and creative category only accounts for about $12.1 \%$. The inconsistent ratio of the business types makes too many net points of the same type concentrated in the original division of the block, while homogeneous conflict of competition is not conducive to the overall image building of the block and the highlight of cultural characteristics. Secondly, the business types are relatively traditional and the participation of tourists is low. Existing historical building development models are mostly based on static visits and purchases. Apart from providing participatory entertainment projects on specific holidays, the lack of normalized experience activities reduces the participation of tourists and is not conducive to satisfying tourists' understanding and experiencing the spiritual needs of the block culture.

2) The development of cultural and creative industry is weak, and the industrial chain is to be formed: Cultural and creative projects in the block mostly exist in solid form and simple display form; there is a lack of in-depth development in three layers including the content layer, monetization layer and derivative layer of the cultural industry chain. Taking derivatives as an example, most cultural and creative derivatives are monotonous in form and simple in function, such as key chains, mobile phone holders and other primary and common cultural and creative products. Cultural and creative products fail to make effective innovation in full combination with the characteristic cultural elements of southern Fujian, which greatly reduces tourists' perception of experience, can not effectively attract tourists, and the return rate is low.

3) The overall fame of the scenic spot is small, and the brand competition is weak: The current brands in the block have not formed prominent strength and had low fame in China. According to survey data, only 59\% of the respondents have seen relevant cultural and tourism propaganda information through $\mathrm{TV}$, WeChat and other common media, and about 50\% said they have a basic understanding of the culture of Five-store Bazaar. The block still has a certain weakness in terms of external publicity and promotion, and it still needs to be further improved in terms of effectively transferring the block image and cultural characteristics to the target group. At the same time, the industry's competitive strengths are not obvious. For example, compared with famous cultural blocks in China, there is a big gap in the cultural industry structure of the block, the management and operation of various businesses, the market information research and judgment mechanism, and the operating capabilities of core companies in the block.

\section{Opportunity analysis}

1) Continuous advancement of "Jinjiang Model": In 2019, Jinjiang's GDP exceeded RMB 250 billion, and the county's economic strength ranked first in the counties of the whole province for 25 consecutive years. In 2019, General Secretary Xi Jinping reiterated the "Jinjiang experience" and highly affirmed the "Jinjiang model" centered on the development of real economy. On the one hand, the continuous advancement of "Jinjiang Model" provides a good material foundation support for the development of the block; on the other hand, as an important external advantage for the development of the block, it is conducive for the block to firstly take the opportunity to integrate the industrialization development of the block's cultural resources into the high-quality development of the regional economy.

2) Promotion of "global tourism" policy: In 2019, the Quanzhou Municipal Government issued the "Quanzhou City Implementation Plan for Accelerating the Development of Global Tourism (2019-2020)". This policy firstly clarifies the current good situation of cultural tourism market, and there is a certain room for improvement. Secondly, it releases a positive signal for supporting and promoting the in-depth development of "global tourism" in various regions of the city, and provides a relatively loose policy environment for the industrialization development of the block of Five-store Bazaar. It is a development opportunity not to be missed for enhancing the linkage and cooperation outside the block, promoting the in-depth integration of culture and tourism, and strengthening the construction of its cultural industry system.

\section{Treats analysis}

1) Fierce regional competition: As far as Fujian is concerned, Jinjiang will inevitably have a "negative effect of tourist corridor phenomenon". The development of cultural tourism in Xiamen, Fuzhou and other places was started relatively early, and various types of tourism supporting facilities are relatively complete. In particular, the overall industrialization of the two historical cultural blocks including Sanfangqixiang in Fuzhou and Gulangyu in Xiamen has formed a relatively mature system. According to survey 
data, when choosing cities in Fujian Province as tourist destinations, about $55.1 \%$ of the respondents chose Xiamen City, and only $16.1 \%$ chose Quanzhou. Fivestore Bazaar has become a "tour and sightseeing transit", which to a certain extent has caused the practical dilemma of Five-store Bazaar to "survive in gap".

2) Limitations of the management system: The government is in a dominant position in the industrialization development of cultural resources. How to straighten out the relationship between cultural undertakings and industries has always been a difficult problem in public cultural governance. Specifically, there has not been a sound cultural industry development supervision and evaluation system, and the traditional top-down evaluation mechanism is still maintained. There are some extreme phenomena of single or multiple supervision bodies so that the evaluation results lack of scientific and democratic natures. On the other hand, the interest coordination mechanism is not perfect, and it is difficult to reach a reasonable distribution of interests in terms of cultural tourism projects involving multiple stakeholders, which in turn affects the unified planning and construction of specific projects. These threats are not conducive to the long-term development of cultural industry in the Fivestore Bazaar.

\section{STRATEGIES FOR INDUSTRIALIZATION DEVELOPMENT OF CULTURAL RESOURCES IN THE FIVE-STORE BAZAAR OF JINJIANG CITY}

Based on the above analysis, this research forms a SWOT matrix strategy diagram, as shown in "Fig. 2". Specifically, it is integrated into the " $3+2$ " model for industrialization development of cultural resources in Five-store Bazaar. "3" refers to the three strategies including planning adjustment, business expansion, and brand establishment. "2" refers to the two basic principles including differentiation and activation.

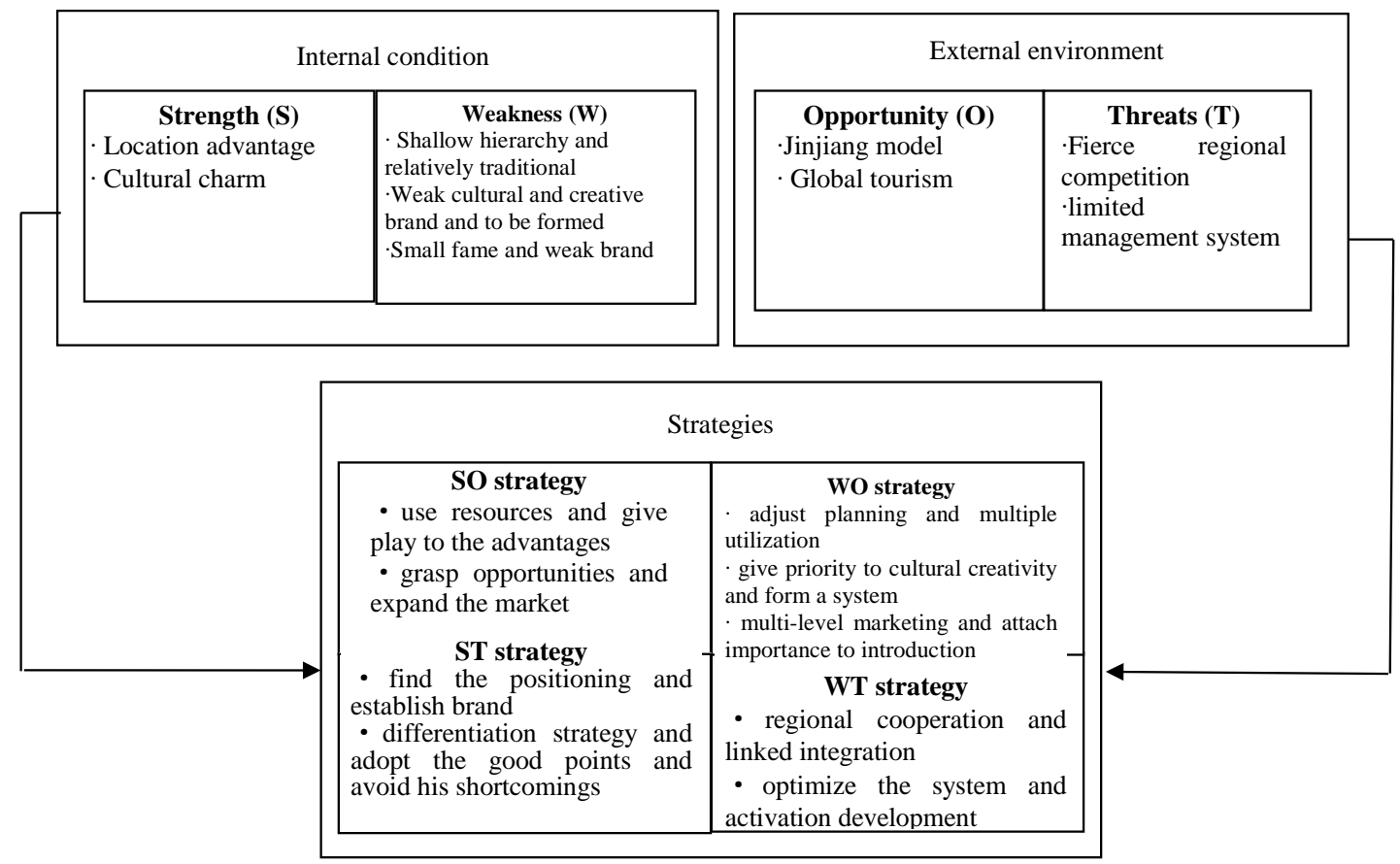

Fig. 2. Schematic diagram of Five-store Bazaar's cultural resources industrialization development strategy.

\section{A. Adjusting the functional planning based on the original appearance of the block}

The first is to maintain the continuation of the original style of the block. On the one hand, it is necessary to continue to implement hierarchical management on existing historical building classifications, follow the principle of integrity, and pay attention to the coordination and harmony with the building community after the occupation of businesses. On the other hand, modern technology should be used to renovate the damaged traditional buildings based on the materials and colors of the original buildings, so as to minimize the impact of artificial marks. The second is to pay attention to 3D space design of the block. Considering from the inside of building, for multi-story buildings and one-story multi-room buildings, the entire building should be utilized to the maximum extent, and the areas within the building should be reasonably divided and transformed to reduce the idleness and waste of available space. From the perspective of 
architecture and the overall appearance of the block, it is needed to pay attention to giving visitors a spatial experience from top to bottom, especially the visual sensory stimulation of the night landscape. Therefore, it is necessary to deal with the application of threedimensional space and the arrangement of sightseeing lines under the limited volume ratio of the block. Finally, it is to highlight the cultural theme of southern Fujian, especially the characteristics of Jinjiang. It is available to use iconic buildings in the block (such as the Cai Family's Temple), combine with the use of specific architectural functions to deeply integrate the communication space, living space and consumption space, making Five-store Bazaar into a new block of Southern Fujian Culture with long history, ancient charm, and modern fashionable appearance.

\section{B. Digging deep into the connotation of resources and expanding diversified businesses}

Digging the connotation of cultural resources is the core content of the industrialization development of the block. The single type of cultural resource will directly lead to problems such as the "long and short legs" of the industrial structure and the gradual slowdown of development momentum in the later period. Therefore, it is necessary to jump out of the current idea of emphasizing traditional construction resources and turn to the combination with other types of cultural resources, especially new cultural businesses. Taking the "intangible cultural heritage + cultural leisure and entertainment service category" as an example, this type of business mainly provides products and services that meet people's spiritual needs. Its integration path includes getting intangible cultural heritage resources with South Fujian characteristics such as Nanyin and puppet puppetry incorporated into various types of space display platforms in the block, such as intangible cultural heritage stage performance venues and experience halls. As for the current "Xianghui Tea House" that belongs to this type in the block, attention should be paid to appropriately increase the frequency of normalized performances, and launch repertoires that meet the needs of daily performances. On the other hand, it is available to take the intangible cultural heritage exhibition hall in Taiping Street in Tianxin District, Changsha, China, as a reference, and use a specific theme "Jinjiang Memory" as the main line to stimulate emotional resonance by reproducing the historical objects, pictures, audio and so on of the "old Jinjiang". In the process of display, consumers are allowed to participate in it, giving visitors a sense of full participative experience and entertainment. In addition, the block can also appropriately introduce popular businesses such as research tourism, health tourism, and tourism performing arts as a supplement to meet the increasingly personalized, diversified and subdivided market demand, and shape the cultural and tourism industry ecology in Five-store Bazaar.

\section{Extending the industrial chain and creating a cultural brand}

A complete cultural industry chain should include the generation of cultural creativity, product development and production, brand building, protection of intellectual property, and overall application and consumption. The "creativity" is the core part of the chain. Five-store Bazaar should focus on this core, and extend the existing cultural industry chain vertically and horizontally to increase the added value of the industry and build a cultural brand. Horizontally, it is needed to strengthen cooperation with other industries. Cultural enterprises in the block can strengthen cooperation with local shoes, apparel, food and other industries in form of business outsourcing and joint product development and production, concentrate on the development of advantageous cultural resources, and gradually form core competitiveness by virtue of the brand effect of Jinjiang to achieve a win-win situation. Vertically, taking animation and film industry in the block as an example, it is available to gradually participate in production, cinema-chain cooperation, animation and film and television derivatives and other links. Architectural elements in the red-brick buildings, such as the dovetail ridge, Minnan red and the like symbols can be extracted and applied to shape the "IP" image. For example, it is possible to draw on the form of study tours and experience tours in Baihuazhou District, Jiangxi Province, to expand traffic and create a unique cultural IP for Five-store Bazaar. In addition, it is also available to pay attention to digging online network cultural characteristics, and grasp online and offline marketing strategies, use the Internet's dividend traffic and comprehensive operating advantages to focus on enhancing the core competitiveness of the block, and break away from the low-end homogenization development dilemma.

\section{Adopting differentiation strategies and integrating regional linkages}

In the industrialization development of Five-store Bazaar, it is also necessary to focus on adopting differentiation strategies and integrating the surrounding cultural resources to face the threats of regional competition. Specifically, the differentiation strategy is to concentrate on the regional cultural resource advantages of southern Fujian, construct exquisite cultural projects, and form core brands. Even if there are the same types of cultural industries as those in the surrounding area, it is also necessary to seek as many differences as possible and be special. It is necessary to make full use of the established platforms in Quanzhou, such as the "Maritime Silk Road: Quanzhou Tourism Resources Trading Platform" and other platforms, establish a combination and joint marketing strategy, use the existing characteristic cultural tourism resources in the ancient towns of Jinjiang City, to realize the complementary advantages 
of different regions, form joint efforts of cultural tourism markets, and improve the overall influence. For example, it is available to draw on the design ideas of Qinhuai District in Nanjing, stand on the construction of Jinjiang's global tourism layout, and form a global tourism spatial pattern dominated by Five-store Bazaar and surrounding cultural and creative parks and extended to the cultural resources of various towns.

\section{E. Adhering to the principle of activation and encouraging public participation}

General Secretary Xi Jinping pointed out, "Let the masses see the mountains and water, and remember the nostalgia". Between the lines, the importance of lively protection of cultural heritage is highlighted. Therefore, the industrialization of cultural resources in the Fivestore Bazaar should be promoted continuously and blended in daily life of local people and give full play to its potential intangible cultural benefits. For cultural resources with insufficient industrialization development conditions in Jinjiang City and narrow range of audiences, the attitude of "holding history and waiting for resurrection" should be adopted to avoid pulling up seedlings to help them grow. In addition, another important manifestation of the live development is to encourage the public to participate in the subsequent construction process of the Five-store Bazaar. In this respect, it is available to learn from the principle of "being people-oriented, organically updated, multi-subject, and joint participation" that Beijing Dashilan followed in its development. Relevant governmental departments and the block-running companies should play the role of social organizations, groups and individuals, and use communities, neighborhood committees and other platforms to arouse the enthusiasm of local people. It is necessary to establish and improve a "policy-society coordination model", and to take advantage of the "Jinjiang experience" to further handle the relationship between tangible channels and intangible channels. For instance, a lively atmosphere can be jointly created by perfecting the government's public culture service mechanism to purchase intangible cultural heritages and the like cultural resources from social forces, and using social organizations to promote the normalization of cultural resource protection activities in the block.

\section{CONCLUSION}

Taking Five-store Bazaar of Jinjiang City as the research object, this paper proposes the " $3+2 "$ model for industrialization development through SWOT analysis from the perspective of industrialization development. At present, the development of the overall cultural resources of the block is still in its infancy. For the industrialization model of cultural resources, promoting the integration of culture and tourism is currently a common measure taken all over China. How to effectively develop cultural and creative industry, fully demonstrate the strength of cultural capital in the region, and avoid falling into the dilemma of low-end homogenization is currently a major challenge faced by the historical block of Five-store Bazaar.

\section{References}

[1] Yang Yuhua, Xu Xuhong. Marxist Economics Interpretation of "Jinjiang Experience" and Countermeasures for High Quality Development [J]. Southeast Academic Research. 2020 (02): 174-182. (in Chinese)

[2] Bunten, Alexis Celeste. Sharing culture or selling out? Developing the commodified persona in the heritage industry[J]. American Ethnologist. 2008, 35(3): 380-395.

[3] Landorf C. A Framework for Sustainable Heritage Management: A Study of UK Industrial Heritage Sites[J] International Journal of Heritage Studies. 2009, 15(6): 494-510.

[4] Chung H, Lee J. Community Cultural Resources as Sustainable Development Enablers: A Case Study on Bukjeong Village in Korea compared with Naoshima Island in Japan[J]. Sustainability. 2019, 11(5): 1401.

[5] Zhang Rui. The foundation, problems of the industrialization of historical and cultural resources of city clusters in the middle reaches of the Yangtze River and the countermeasures [J]. Theory Monthly. 2020 (01): 101-109. (in Chinese)

[6] Chen Xueqi, Xia Senwei, Yang Yang. Social and Economic benefits of Protection and Development of Traditional Historic and Cultural blocks - Jinjiang wudianshi traditional blocks as an example [J]. Fujian Architecture Construction. 2019 (02): 14-17. (in Chinese)

[7] Yin Ziyan, Huang Anmin. Research on Tourist Experience of Cultural and Creative Block Based on Web Text Analysis Take Wudian of Jinjiang as an Example [J]. Journal of Minjiang University. 2019, 40 (04): 85-92. (in Chinese)

[8] Zhang Chunxiang. The Evaluation of Industrial Competitiveness of Regional Cultural Tourism Based on the Diamond Model [J]. Chinese Journal of Management. 2018, 15 (12): 1781-1788. (in Chinese)

[9] Zhang Haihong, Zhang Yun. Research on the Evaluation of Tourism Industry Competitiveness in Western Hunan Based on Structural Equation Model [J]. Journal of Jishou University (Social Science Edition). 2014, 35 (S2): 32-35. (in Chinese)

[10] Yu Junqu, Qin Hongzeng. A study of the village-based mechanism of inheritance and innovation of traditional rural cultural resources and rural vitalization $[\mathrm{J}]$. Journal of Yunnan Nationalities University (Philosophy and Social Sciences Edition). 2019, 36 (03): 63-69. (in Chinese)

[11] Zhang X. Research on the Development Strategies of Rural Tourism in Suzhou Based on SWOT Analysis[J]. Energy Procedia. 2012, 16: 1295-1299.

[12] Jin Liang, Chen Shixiang. Cultural Attribute "Trichotomy" and Construction of China's Public Cultural Governance [J]. Journal of Shanghai Jiaotong University (Philosophy and Social Sciences Edition). 2018, 26 (02): 76-85. (in Chinese) 